\title{
Construction of Multiple-Rate QC-LDPC Codes Using Hierarchical Row-Splitting
}

\author{
Peiyao Zhao, Zhaocheng Wang, and Qi Wang
}

\begin{abstract}
In this letter, we propose an improved method called hierarchical row-splitting with edge variation for designing multiple-rate quasi-cyclic low-density parity-check (QC-LDPC) codes, which constructs lower-rate codes from a high-rate mother code by row-splitting operations. Consequently, the obtained QC-LDPC codes with various code rates have the same blocklength and can share common hardware resources to reduce the implementation complexity. Compared with the conventional row-combining-based algorithms, a wider range of code rates are supported. Moreover, each individual rate code could be separately optimized, making it easier to find a set of multiplerate QC-LDPC codes with good performance for all different rates. Simulation results demonstrate that the obtained codes outperform the counterparts from digital video broadcastingsecond generation terrestrial.
\end{abstract}

Index Terms-Low density parity check (LDPC) codes, multiple-rate, quasi-cyclic, row-splitting.

\section{INTRODUCTION}

$\mathbf{P}$ RACTICAL communication systems have to support various code rates for different user scenarios, with the same encoder/decoder pair shared for the whole set of codes, which is referred to as rate-compatibility. The design of ratecompatible (RC) low-density parity-check (LDPC) codes has been widely investigated using code modifying techniques such as puncturing, extending, and combining [1]-[7].

Many previous works focus on protograph-based LDPC codes [1]-[4]. These codes are built from a small graph known as a protograph by "copy-and-permute" operations and enjoy the advantages of near-capacity performance and low encoding/decoding complexity. The protograph-based RC LDPC codes construction is studied extensively by Divsalar et al. in [1], which yields both the low decoding thresholds and the linear minimum distance growth. Protograph-based raptor-like LDPC codes are constructed in [2] by sequentially adding degree-one variable nodes to a well-designed highest-rate code. Nguyen and Nosratinia [3], [4] introduce an extending technique by adding the same number of columns and rows into the check matrix of a high-rate code, and consequently a nested structure could be obtained. The RC LDPC codes constructed

Manuscript received September 2, 2015; revised February 21, 2016 and March 31, 2016; accepted April 9, 2016. Date of publication April 13, 2016; date of current version June 8, 2016. This work was supported in part by National Natural Science Foundation of China (Grant No. 61271266), in part by Beijing Natural Science Foundation (Grant No. 4142027), in part by Shenzhen Peacock Plan (No. 1108170036003286), and in part by Shenzhen Visible Light Communication System Key Laboratory (ZDSYS20140512114229398). The associate editor coordinating the review of this letter and approving it for publication was M. Baldi.

The authors are with the Tsinghua National Laboratory for Information Science and Technology, Department of Electronic Engineering, Tsinghua University, Beijing 100084, China (e-mail: zhaopy14@mails.tsinghua.edu.cn; zcwang@tsinghua.edu.cn; qiwang11@mails.tsinghua.edu.cn).

Digital Object Identifier 10.1109/LCOMM.2016.2553658 from the above methods share the same information blocklength, which makes them are suitable for hybrid automatic repeat request (HARQ) applications.

Another common way of generating RC LDPC codes is puncturing, that is selectively discarding some parity bits from a low rate code to achieve higher rate codes. A two-phase puncturing algorithm is proposed in [5] by evaluating the number of short cycles with low approximate cycle extrinsic message degree. Zho et al. [6] further considers the novel concept of completely punctured cycle trapping sets to calculate the best puncturing pattern. However, puncturing reduces the code blocklength, which degrades performance when compared to an LDPC code with the original blocklength.

To maintain a constant blocklength, Casado et al. presented the row-combining with edge variation (RCEV) algorithm to construct multiple-rate LDPC codes [7], where the check matrices are obtained by combining rows of a low-rate mother code, and some edges are added or deleted to match the desired degree distributions. Nevertheless, the strongly coupled feature of the method makes it difficult to find a set of codes with good performance for all rates, and the supported rates are also restricted.

In this letter, we propose a new hierarchical row-splitting with edge variation (HRSEV) algorithm to generate a set of multiple-rate QC-LDPC codes by splitting the rows of a highrate mother code, which is a much more flexible approach than the row-combining based algorithms. In addition, unequal ' 1 '-component distributions in the row-splitting are also proposed, such that a wider range of code rates can be supported, and a large number of candidate codes can be searched to optimize the performance of the constructed codes. Moreover, each rate code can be optimized separately due to the loosely coupled feature and hierarchical process of our proposed algorithm. Simulation results demonstrate that our proposed algorithm is capable of constructing a set of multiple-rate QC-LDPC codes with good performance for all different rates.

\section{QC-LDPC CODES}

QC-LDPC codes are a class of LDPC codes whose parity check matrix is an array of square sub-matrices each with size $b \times b$ [8], where the sub-matrix is a null matrix or a circulant matrix. Denote $m$ and $n$ as the number of rows and columns of the parity check matrix, respectively. We define $\mathbf{H}_{\text {base }}$ with size $M \times N$ as the base matrix of QC-LDPC codes by replacing null matrices with zeros and circulant matrices with ones, where $M=m / b$ and $N=n / b$. Note that the row-splitting operations for QC-LDPC codes are performed on the rows of sub-matrices instead of individual rows to maintain the regularity of check matrices, which is equivalent to performing row-splitting on the rows of base matrices. 
The Reed-Solomon progressive edge growth (RS-PEG) algorithm [11] is capable of constructing QC-LDPC codes with large girth under certain degree distribution, which plays an essential role on the performance of QC-LDPC codes. Extrinsic information transfer (EXIT) charts [9] are employed to optimize the degree distributions that are associated with each code rate. However, the general EXIT charts cannot be applied to compute the decoding thresholds of QC-LDPC codes with the same degree distribution. Thus we adopt the PEXIT method proposed in [10] to compute the thresholds of QC-LDPC codes with certain base matrices. The decoding threshold is defined as the minimum channel quality that supports reliable decoding when the blocklength is asymptotically large. Consequently, it can be used to evaluate the performance of the constructed QC-LDPC codes in water-fall regions instead of full simulations to reduce the computational complexity.

\section{Construction of Multiple-Rate QC-LDPC CODES}

\section{A. Row-Splitting Operation}

Row-splitting operation replaces each single row with several rows, such that the obtained rows can be combined to the original one in reverse. For example, the parity check matrix of a rate- $9 / 10$ code is given by

$$
\mathbf{H}_{9 / 10}=\left(\begin{array}{llllllllll}
0 & 1 & 1 & 0 & 1 & 0 & 1 & 1 & 0 & 1
\end{array}\right) .
$$

A rate- $4 / 5$ code can be constructed by splitting the row of $\mathbf{H}_{9 / 10}$ into two rows and distributing the ' 1 '-components equally to the new rows. Note that we only consider checkregular codes [9], i.e., all rows have almost the same degrees, as non-concentrated row degree distributions may degrade the performance of LDPC codes. Due to the flexibility of row-splitting, there are many different ways to split rows. Consequently, a large number of candidate codes can be searched, which makes it easy to find a well-performed one. One example of the obtained rate- $4 / 5$ code is expressed by

$$
\mathbf{H}_{4 / 5}=\left(\begin{array}{cccccccccc}
0 & 1 & 0 & 0 & 0 & 0 & 1 & 1 & 0 & 0 \\
0 & 0 & 1 & 0 & 1 & 0 & 0 & 0 & 0 & 1
\end{array}\right) .
$$

However, equal ' 1 '-components distribution in the row-splitting limits the supported rates, which means that the rows number of the obtained lower-rate code must be an integral multiple of the high-rate mother code. In order to extend the supported rates, we propose unequal ' 1 '-components distribution in the row-splitting operation. For instance, we can split each row of $\mathbf{H}_{4 / 5}$ into two rows and distribute $2 / 3$ of the ' 1 '-components to one row and $1 / 3$ of the ' 1 '-components to the other row. The result is shown in $\mathbf{H}_{7 / 10}^{\mathrm{pre}}$, where the rows with smaller degrees are emphasized.

$$
\mathbf{H}_{7 / 10}^{\mathrm{pre}}=\left(\begin{array}{cccccccccc}
0 & 1 & 0 & 0 & 0 & 0 & 0 & 1 & 0 & 0 \\
0 & 0 & 0 & 0 & 0 & 0 & 1 & 0 & 0 & 0 \\
0 & 0 & 0 & 0 & 1 & 0 & 0 & 0 & 0 & 0 \\
0 & 0 & 1 & 0 & 0 & 0 & 0 & 0 & 0 & 1
\end{array}\right) .
$$

Then, each two rows with smaller degrees are combined together to obtain a rate-7/10 code given by

$$
\mathbf{H}_{7 / 10}=\left(\begin{array}{cccccccccc}
0 & 1 & 0 & 0 & 0 & 0 & 0 & 1 & 0 & 0 \\
0 & 0 & 0 & 0 & 1 & 0 & 1 & 0 & 0 & 0 \\
0 & 0 & 1 & 0 & 0 & 0 & 0 & 0 & 0 & 1
\end{array}\right) .
$$

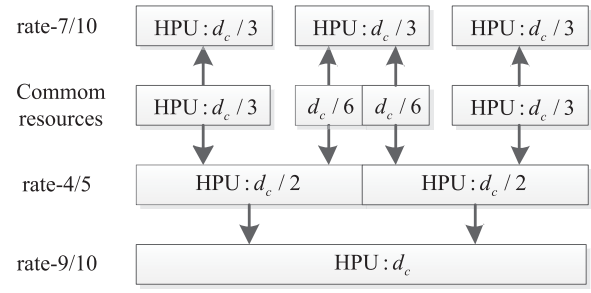

Fig. 1. HPU architecture of the proposed multiple-rate LDPC codes.

As we can see, the row-splitting operation with unequal ' 1 '-components distribution firstly generates some rows with smaller degrees, which are then combined to guarantee concentrated row degree distribution of the obtained code. Therefore, a wide range of rates can be supported in comparison to the previous row-combining based algorithms. Moreover, the obtained multiple-rate codes are loosely coupled. Specifically, the construction of lower-rate codes does not influence the already constructed higher-rate codes. This feature makes it possible to optimize each rate code separately. Besides that, the row-splitting operations do not generate new cycles, referred to as the cycle free feature. Such that the girths of the constructed lower-rate codes are no less than that of the original high-rate mother code.

The multiple-rate LDPC codes constructed by row-splitting operation can share common hardware resources to reduce the hardware implementation complexity. A typical LDPC decoder adopting sum-product algorithm [12] includes horizontal process units (HPUs) for horizontal process and vertical process units (VPUs) for vertical process. Obviously, VPUs can be shared by all rate codes since the row-splitting operations do not change the column degrees. Meanwhile, HPUs of higher-rate decoder can be achieved by grouping some specific HPUs of lower-rate decoder. For example, the HPU architecture of the above rate-9/10, 4/5, 7/10 codes is illustrated in Fig. 1, where $d_{c}$ denotes the row degree of the highest rate-9/10 code.

\section{B. Improved Multiple-Rate QC-LDPC Codes Construction}

In this section we propose an improved method for constructing multiple-rate QC-LDPC codes. Since the lower-rate code is generated from the previous higher-rate code by row-splitting and edge variation is introduced to optimize the column degree distributions, the obtained codes are called hierarchical row-splitting with edge variation codes.

Assuming that $K$ different rates $r_{k}, 1 \leq k \leq K$ are desired in descending order. The corresponding base matrix of each rate code is denoted as $\mathbf{H}_{r_{k}}$. By adopting row-splitting operation with unequal ' 1 '-components distribution, the intermediate matrix of each rate code is denoted as $\mathbf{H}_{r_{k}}^{\text {pre }}$. Furthermore, $S_{r_{i} \rightarrow r_{i+1}}, 1 \leq i \leq K-1$ is defined as the row-splitting operation that transforms $\mathbf{H}_{r_{i}}^{\text {pre }}$ into $\mathbf{H}_{r_{i+1}}^{\mathrm{pre}}$.

Firstly, the degree distributions of desired rates are optimized employing EXIT charts [9]. Then the highest-rate code, i.e. rate- $r_{1}$ code, is constructed using RS-PEG [11] algorithm which ensures that the obtained code has a large girth and a low error floor. The base matrix of the obtained rate- $r_{1}$ QC-LDPC code $\mathbf{H}_{r_{1}}$ has constant row degree, denoted as $d_{c_{1}}$. Since row-splitting and row-combining operations do not 
change the number of ' 1 ' components in the base matrices, the desired row degree of rate- $r_{k}$ code is calculated by

$$
d_{c_{k}}=\frac{1-r_{1}}{1-r_{k}} d_{c_{1}}, \quad 1 \leq k \leq K
$$

Initially, $\mathbf{H}_{r_{1}}^{\mathrm{pre}}$ is set the same as $\mathbf{H}_{r_{1}}$. Then operation $S_{r_{1} \rightarrow r_{2}}$ is applied to $\mathbf{H}_{r_{1}}^{\text {pre }}$ to get the next intermediate matrix $\mathbf{H}_{r_{2}}^{\text {pre }}$. As stated before, the method of splitting a certain row of $\mathbf{H}_{r_{1}}^{\text {pre }}$ depends on the relationship between its row degree $d$ and the desired row degree of rate- $r_{2}$ code. Assuming that

$$
\frac{d}{d_{c_{2}}}=p+q, p \in \mathbb{Z}, \quad 0 \leq q<1,
$$

where $p$ and $q$ is the integral part and decimal part, respectively. If $d$ is no more than $d_{c_{2}}$, i.e. $p+q \leq 1$, the considered row remains unchanged. However, if $d$ is an integral multiple of $d_{c_{2}}$, i.e. $p>0, q=0$, the considered row is split into $p$ rows and the ' 1 '-components are distributed equally to the new rows. Furthermore, if $p>0, q \neq 0$, unequal ' 1 '-components distribution is employed. Specifically, the considered row is split into $p+1$ rows, among which $p$ rows have the degree $d_{c_{2}}$ and one row has the degree $q d_{c_{2}}$. After the intermediate matrix $\mathbf{H}_{r_{2}}^{\mathrm{pre}}$ is obtained, the rows with degrees less than $d_{c_{2}}$ are combined to guarantee concentrated row degree distribution of the base matrix $\mathbf{H}_{r_{2}}$. Since the row-splitting and row-combining operations do not change the column degree distribution, edge variation [7] is then introduced to match the optimal column degree distribution of each rate code by adding or deleting some ' 1 '-components on the base matrix.

Due to the cycle free feature of row-splitting operations and the large girth of the highest-rate code obtained by using RS-PEG algorithm, the error floor behavior of the constructed multiple-rate codes is generally satisfactory. As a result, we mainly focus on the performance in water-fall regions, which can be efficiently evaluated by the decoding threshold using PEXIT charts analysis [10]. Generally, a decoding threshold with gap to capacity less than $0.25 \mathrm{~dB}$ is already a good result. In practice, we could also generate a number of candidate codes that satisfy the decoding threshold constraints and select the best one by verifying the performance in both water-fall and error floor regions through full simulations.

The flexibility of row-splitting operations enables us to construct multiple-rate QC-LDPC codes of desired rates one by one from the highest rate to the lowest rate. The latter operation $S_{r_{i} \rightarrow r_{i+1}}, 1<i<K$ generates $\mathbf{H}_{r_{i+1}}^{\mathrm{pre}}$ from the intermediate matrix of higher-rate code $\mathbf{H}_{r_{i}}^{\text {pre }}$ in the same way as $S_{r_{1} \rightarrow r_{2}}$. Then $\mathbf{H}_{r_{i+1}}$ is obtained through combining the rows of $\mathbf{H}_{r_{i+1}}^{\mathrm{pre}}$ with degrees less than $d_{c_{i+1}}$ and edge variation is applied to match the optimal column degree distribution of rate- $r_{i+1}$ code. Fig. 2 shows the splitting process of constructing a series of multiple-rate QC-LDPC codes, in which the number denotes the distribution of ' 1 '-components in the row-splitting and the box denotes the row-combining operation.

Note that the code parameters including code rates and submatrix size should be carefully designed, among which the sub-matrix size is selected according to [11]. Furthermore,

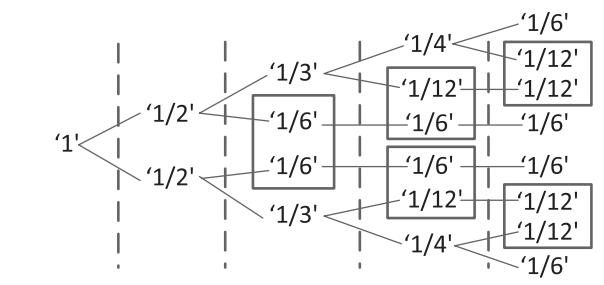

rate- $9 / 10$ rate- $4 / 5$ rate- $7 / 10$ rate- $3 / 5$ rate- $2 / 5$

Fig. 2. An example of the hierarchical splitting process.

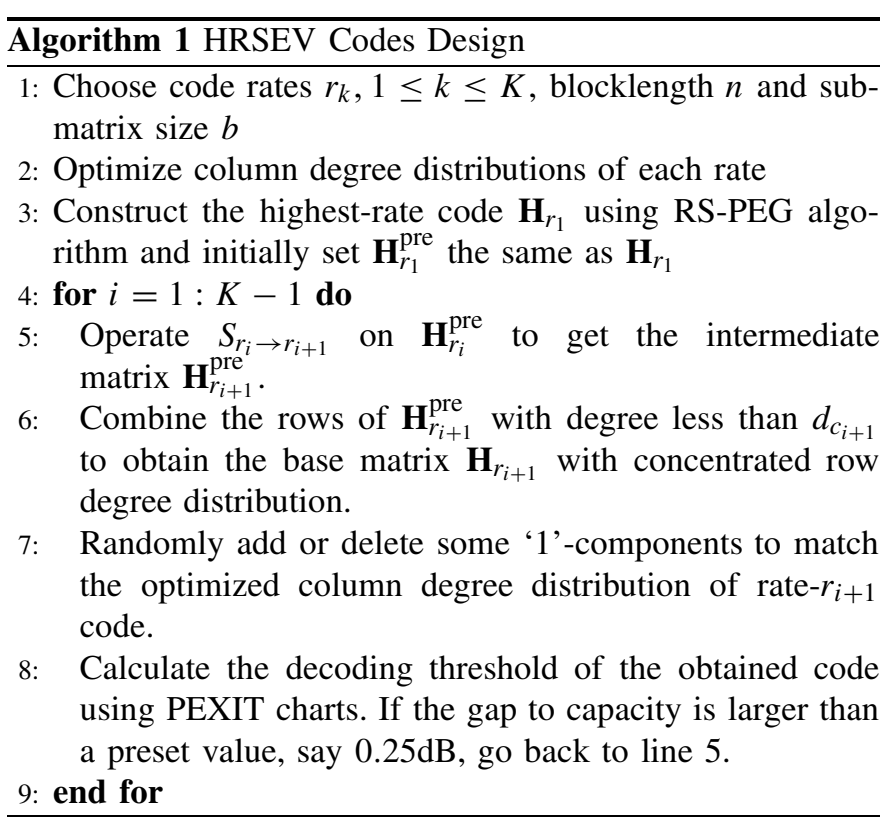

we propose a criterion, called the level of fragmentation, to evaluate if the rates set is suitable for the proposed hierarchical row-splitting algorithm, which is defined as

$$
\alpha=\frac{d_{c_{K}}}{\operatorname{gcd}\left(d_{c_{1}}, d_{c_{2}}, \ldots, d_{c_{K}}\right)}
$$

where $\operatorname{gcd}\left(d_{c_{1}}, d_{c_{2}}, \ldots, d_{c_{K}}\right)$ denotes the greatest common divisor of $d_{c_{k}}, 1 \leq k \leq K$, and $d_{c_{k}}$ is calculated by $\mathrm{Eq}(5)$. Large $\alpha$ indicates that HPUs are split into small fragments, which leads to high hardware complexity and may degrade the performance of lower-rate codes as well. Generally, the level of fragmentation $\alpha$ should be no larger than 2 .

The design procedure of multiple-rate HRSEV QC-LDPC codes is summarized in Algorithm 1.

\section{Simulation Results}

A series of multiple-rate QC-LDPC codes with target rates of $\{0.4,0.6,0.8\}$, code length $n=61440$ and sub-matrix size $b=512$ were constructed. The optimized column degree distribution of each code rate utilizing the EXIT charts analysis is shown in Table. I.

Table. II gives the decoding thresholds of the constructed HRSEV codes. The decoding thresholds were computed by PEXIT charts [10], showing that the gaps to capacity between $0.22 \mathrm{~dB}$ and $0.26 \mathrm{~dB}$ have been achieved. 
TABLE I

Column Degree Distribution of Each Code Rate

\begin{tabular}{c|c|c|c|c}
\hline \hline \multirow{2}{*}{ Rate } & \multicolumn{4}{|c}{ Column degree value } \\
\cline { 2 - 5 } & 12 & 11 & 3 & 2 \\
\hline 0.4 & 16 & 0 & 32 & 72 \\
0.6 & 18 & 0 & 58 & 44 \\
0.8 & 0 & 19 & 76 & 25 \\
\hline
\end{tabular}

TABLE II

DECODING THRESHOLDS OF THE CONSTRUCTED Multiple-RATE QC-LDPC CODES

\begin{tabular}{c|c|c|c}
\hline \hline Rate & Decoding Thres. (dB) & Capacity (dB) & Gap to Capacity \\
\hline 0.4 & 0.021 & -0.238 & 0.259 \\
0.6 & 0.902 & 0.679 & 0.223 \\
0.8 & 2.274 & 2.040 & 0.234 \\
\hline \hline
\end{tabular}

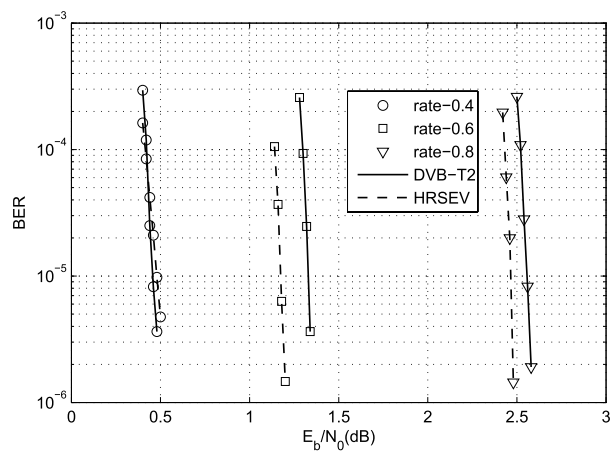

Fig. 3. BER performance comparison of the proposed HRSEV codes and the DVB-T2 LDPC codes over AWGN channel.

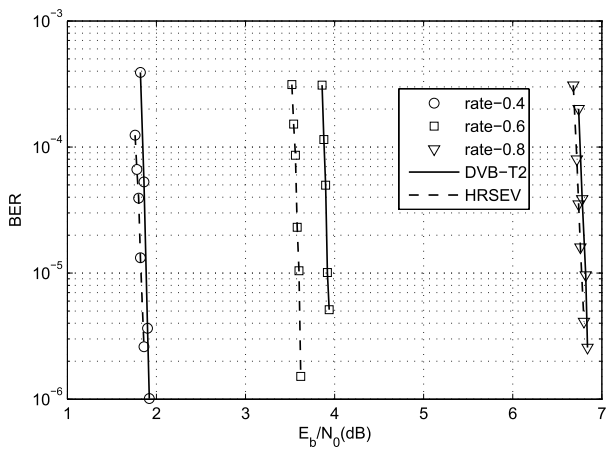

Fig. 4. BER performance comparison of the proposed HRSEV codes and the DVB-T2 LDPC codes over Rayleigh channel.

Simulations were also carried out to evaluate the performance of the obtained HRSEV codes. The corresponding codes with the same rates in DVB-T2 [13], whose blocklength is 64800 , were employed as a comparison. Fig. 3 and 4 compare the bit error rate (BER) performance over the additive white gaussian noise (AWGN) channel and Rayleigh channel with BPSK modulation, respectively, in which the sum-product algorithm was adopted at the LDPC decoder and the maximum iteration number was set to 50 .

As illustrated in Fig. 3, at BER level of $10^{-5}$, the obtained HRSEV codes provide approximately $0.15 \mathrm{~dB}$ and 0.09 $\mathrm{dB}$ gains over those counterparts from DVB-T2 for the rate 0.6 and 0.8 , respectively. Additionally, we also notice that for the rate 0.4 , the BER performance of these two kinds of codes is nearly the same. Fig. 4 shows that the obtained HRSEV codes exhibit approximately $0.32 \mathrm{~dB}$ gains over the LDPC codes from DVB-T2 for the rate 0.6, at BER level of $10^{-5}$. Moreover, for the other two rates, our proposed HRSEV codes also perform better.

It should be noted that the blocklength of the LDPC codes from DVB-T2 is actually larger than the HRSEV codes. As the HRSEV codes achieve better performance with a smaller blocklength in comparison to the DVB-T2 codes, we can claim that our proposed method is capable of constructing multiple-rate QC-LDPC with good performance.

\section{CONCLUSIONS}

An improved method for designing multiple-rate QC-LDPC codes is proposed, whereby the row-splitting operation is utilized to construct lower-rate codes from a high-rate mother code, such that common hardware resources can be shared by all rate codes to simplify the implementation complexity. In comparison to the conventional row-combining based algorithms, a wider range of code rates are supported and the flexibility of row-splitting enables the performance of each rate code be optimized separately. Simulation results demonstrate that the proposed algorithm is capable of constructing multiple-rate QC-LDPC codes with good performance.

\section{REFERENCES}

[1] D. Divsalar, S. Dolinar, C. R. Jones, and K. Andrews, "Capacityapproaching protograph codes," IEEE J. Sel. Areas Commun., vol. 27, no. 6, pp. 876-888, Aug. 2009

[2] T.-Y. Chen, K. Vakilinia, D. Divsalar, and R. D. Wesel, "Protographbased raptor-like LDPC codes," IEEE Trans. Commun., vol. 63, no. 5, pp. 1522-1532, May 2015.

[3] T. V. Nguyen, A. Nosratinia, and D. Divsalar, "The design of ratecompatible protograph LDPC codes," IEEE Trans. Commun., vol. 60 , no. 10, pp. 2841-2850, Oct. 2012.

[4] T. V. Nguyen and A. Nosratinia, "Rate-compatible short-length protograph LDPC codes," IEEE Commun. Lett., vol. 17, no. 5, pp. 948-951, May 2013.

[5] R. Asvadi and A. H. Banihashemi, "A rate-compatible puncturing scheme for finite-length LDPC codes," IEEE Commun. Lett., vol. 17, no. 1, pp. 147-150, Jan. 2013.

[6] H. Zhou, D. G. M. Mitchell, N. Goertz, and D. J. Costello, Jr., "Robust rate-compatible punctured LDPC convolutional codes," IEEE Trans. Commun., vol. 61, no. 11, pp. 4428-4439, Nov. 2013.

[7] A. I. V. Casado, W.-Y. Weng, S. Valle, and R. D. Wesel, "Multiple-rate low-density parity-check codes with constant blocklength," IEEE Trans. Commun., vol. 57, no. 1, pp. 75-83, Jan. 2009.

[8] L. Chen, J. Xu, I. Djurdjevic, and S. Lin, "Near-Shannon-limit quasicyclic low-density parity-check codes," IEEE Trans. Commun., vol. 52, no. 7, pp. 1038-1042, Jul. 2004.

[9] S. ten Brink, G. Kramer, and A. Ashikhmin, "Design of lowdensity parity-check codes for modulation and detection," IEEE Trans. Commun., vol. 52, no. 4, pp. 670-678, Apr. 2004.

[10] G. Liva and M. Chiani, "Protograph LDPC codes design based on EXIT analysis," in Proc. IEEE Global Telecommun. Conf., Washington, DC, USA, Nov. 2007, pp. 3250-3254.

[11] I. Djurdjevic, J. Xu, K. Abdel-Ghaffar, and S. Lin, "A class of lowdensity parity-check codes constructed based on Reed-Solomon codes with two information symbols," IEEE Commun. Lett., vol. 7, no. 7, pp. 317-319, Jul. 2003.

[12] Z. Cui, Z. Wang, and Y. Liu, "High-throughput layered LDPC decoding architecture," IEEE Trans. Very Large Scale Integr. (VLSI) Syst., vol. 17, no. 4, pp. 582-587, Apr. 2009.

[13] Digital Video Broadcasting (DVB): Frame Structure, Channel Coding and Modulation for a Second Generation Digital Terrestrial Television Broadcasting System (DVB-T2), European Telecommunications Standards Institute (ETSI), France. 